\title{
TOWARDS GUIDED UNDERWATER SURVEY USING LIGHT VISUAL ODOMETRY
}

\author{
M.M. Nawaf *, P. Drap, J.P. Royer, D. Merad, M. Saccone \\ Aix-Marseille Université, CNRS, ENSAM, Université De Toulon, LSIS UMR 7296, \\ Domaine Universitaire de Saint-Jérôme, Bâtiment Polytech, Avenue Escadrille Normandie-Niemen, 13397, Marseille, France. \\ mohamad-motasem.nawaf@univ-amu.fr
}

\section{Commission II}

KEY WORDS: Visual odometry, underwater imaging, computer vision, probabilistic modelling, embedded systems.

\begin{abstract}
:
A light distributed visual odometry method adapted to embedded hardware platform is proposed. The aim is to guide underwater surveys in real time. We rely on image stream captured using portable stereo rig attached to the embedded system. Taken images are analyzed on the fly to assess image quality in terms of sharpness and lightness, so that immediate actions can be taken accordingly. Images are then transferred over the network to another processing unit to compute the odometry. Relying on a standard ego-motion estimation approach, we speed up points matching between image quadruplets using a low level points matching scheme relying on fast Harris operator and template matching that is invariant to illumination changes. We benefit from having the light source attached to the hardware platform to estimate a priori rough depth belief following light divergence over distance low. The rough depth is used to limit points correspondence search zone as it linearly depends on disparity. A stochastic relative bundle adjustment is applied to minimize re-projection errors. The evaluation of the proposed method demonstrates the gain in terms of computation time w.r.t. other approaches that use more sophisticated feature descriptors. The built system opens promising areas for further development and integration of embedded computer vision techniques.
\end{abstract}

\section{INTRODUCTION}

Mobile systems nowadays undergo a growing need for selflocalization task to accurately determine its absolute/relative position over time. Despite the existence of very efficient technologies for this purpose that can be used on-ground (indoor/outdoor) and in-air such as Global Positioning System (GPS), optical, radio beacons, etc. However, in the underwater context most of these signals are jammed so that the corresponding techniques cannot be used. On the other side, solutions based on active acoustics such as imaging sonars and Doppler Velocity Logs (DVL) devices remains expensive and requires high technical skills for deployment and operation. Moreover, their size specifications prevents their integration within small mobile systems or even being hand held. The research for an alternative is ongoing, notably, the recent advances in embedded systems outcomes relatively small, powerful and cheap devices. This opens interesting perspectives to adapt a light visual odometry approach that provides relative path in real-time, which describes our main research direction. The developed solution is integrated within underwater archaeological site survey task where it plays an important role to facilitate image acquisition. An example of targeted underwater site is shown in Figure 1.

In underwater survey tasks, mobile underwater vehicles (or divers) navigate over the target site to capture images. The obtained images are treated in a later phase to obtain various information and also to form a realistic 3D model using photogrammetry techniques (Drap, 2012). In such a situation, the main problem is to totally cover the underwater site before ending the mission. Otherwise, we may obtain incomplete 3D models and the mission cost will raise significantly as further exploitation is needed. However, the absence of an overall view of the site es-

${ }^{*}$ Corresponding author

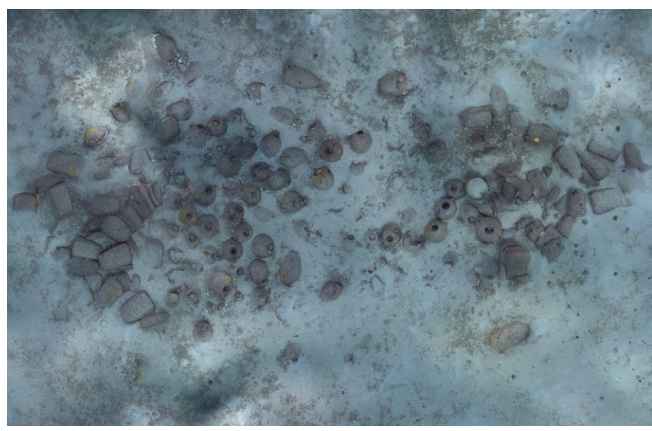

(a) Overall orthphoto

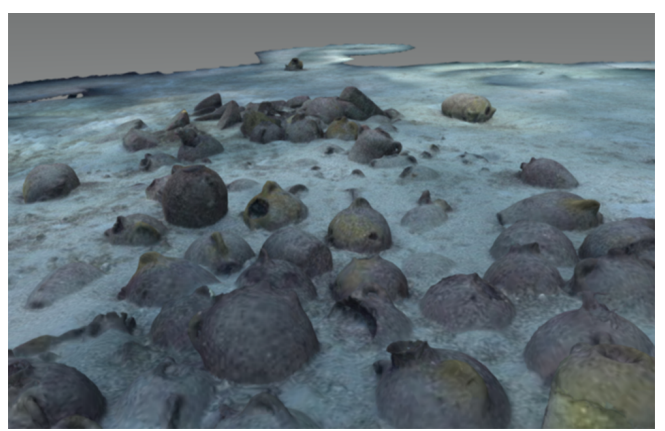

(b) Close-up view

Figure 1. Example of a 3D model of an underwater site that is a Phoenician shipwreck recently discovered located near Malta.

pecially under bad lighting conditions makes the scanning operation blind. In practice, this yields to over-scanning the site which is a waste of time and cost. Moreover, the quality of the taken images may go below an acceptable limit. This mainly happens in terms of lightness and sharpness, which is often hard to 
quantify visually on the fly. In this work, we propose solutions for the aforementioned problems. Most importantly, we propose to guide the survey based on a visual odometry approach that runs on a distributed embedded system in real-time. The output ego-motion helps to guide the site scanning task by showing approximate scanned areas. Moreover, an overall subjective lightness indicator is computed for each image to help controlling the lighting and the distance to target which prevents going too dark or overexposed. Similarly, an image sharpness indicator is also computed to avoid having blurred images, and when necessary, to slow down the motion. Overall, we provide a complete hardware and software solution for the problem.

In common approaches of visual odometry, a significant part of the overall processing time is spent on feature points detection, description and matching. In the tested baseline algorithm inspired from (Kitt et al., 2010), the aforementioned operations represent $\sim 65 \%$ of processing time in case of local/relative bundle adjustment (BA) approach, which occupies in return the majority of the time left. Despite their accuracy and successful wide applications, modern features descriptors such as SIFT (Lowe, 2004) and SURF (Bay et al., 2006) rely on differences of Gaussians (DoG) and fast Hessian respectively for feature detection. This is two times slower than the traditional Harris detector (Gauglitz et al., 2011). Further, the sophisticated descriptors that are invariant to scale and rotation, which is not necessary in stereo matching, slow down the computation. And finally, a brute force matching is often used which is also a time consuming. In our proposed method, we rely on low level Harris based detection and template matching procedure. Whereas in traditional stereo matching the search for correspondence is done along the epipolar line within certain fixed range, in our method we proceed first by computing a priori rough depth belief based on image lightness and following the low of light divergence over distance. This is only valid in a configuration where light sources are fixed to the stereo camera rig, which is the case in our designed system. Our contribution is that we benefit from the rough depth estimation to limit points correspondence search zone since the stereo disparity depends linearly on the distance. The search zone is defined by a preliminary learning phase as will be seen later.

A traditional approach to compute visual odometry based on image quadruplets (two distant captures of a stereo image pair) suffers from rotation and translation drifts that grows with time (Kitt et al., 2010). The same effect applies to the methods based on local BA (Mouragnon et al., 2009). In contrary, the solutions that are based on using features from the entire image set, such as global BA (Triggs et al., 2000), requires more computational resources which are very limited in our case. Similarly, the simultaneous localization and mapping (SLAM) approaches (Thrun et al., 2005), which are known to perform good loop closure, are highly computationally intensive especially when complex particle filters are used (Montemerlo and Thrun, 2007). So they can only operate in moderately sized environments if real-time processing is needed. In our method, we adopt a relative BA method proposed in (Nawaf et al., 2016), which proceed in the same way as local method in optimizing a subset of image frames. However, it differs in the way of selecting the frames subset, as local method uses Euclidean distance and deterministic pose positions to find the $k$ closest frames, the relative method represents the poses in a probabilistic manner, and uses a divergence measure to select such sub set.

The rest of the paper is organized as follows: We survey related works in Section 2. In Section 3 we describe the designed hard- ware platform that we used to implement our solution. Section 4 presents image quality measurement algorithms. Our proposed visual odometry method is explained in Section 5. The analytical results are verified through simulation experiments presented in Section 6. Finally, in Section 7, we present a summary and conclusions. Few parts of this work have been presented in (Nawaf et al., 2016).

\section{RELATED WORKS}

\subsection{Feature Points Matching}

Common ego-motion estimation methods rely on feature points matching between several poses (Nistér et al., 2004). The choice of the used approach for matching feature points depends on the context. For instance, features matching between freely taken images ( 6 degrees of freedom), has to be invariant to scale and rotation changes. Scale invariant feature descriptors (SIFT) (Lowe, 2004) and the Speeded Up Robust Features (SURF) (Bay et al., 2006) are well used in this context (Nawaf and Trémeau, 2014). In this case, the search for a point's correspondence is done w.r.t. all points in the destination image.

In certain situations, some constraints can be imposed to facilitate the matching procedure. In particular, limiting the correspondence search zone. For instance, in case of pure forward motion, the focus of expansion (FOE) being a single point in the image, the search for the correspondence for a given point is limited to the epipolar line (Yamaguchi et al., 2013). Similarly, in case of sparse stereo matching the correspondence point lies on the same horizontal line in case of rectified stereo or on the epipolar line otherwise. This speeds up the matching procedure first by having less comparisons to perform, and second low-level features can be used (Geiger et al., 2011). According to our knowledge there is no method that proposes an adaptive search range following a rough depth estimation from lightness in underwater imaging.

\subsection{Ego-Motion Estimation}

Estimating the ego-motion of a mobile system is an old problem in computer vision. Two main categories of methods are developed in parallel, namely; simultaneous localization and mapping (SLAM) (Davison, 2003), and visual odometry (Nistér et al., 2004). In the following we highlight the main steps for both approaches as well as hybrid solutions trying to combine their advantages.

SLAM family of methods uses probabilistic model to handle vehicle pose, although this kind of methods is developed to handle motion sensors and map landmarks, they work efficiently with visual information solely. In this case, a map of the environment is built and at the same time it is used to deduce the relative pose, which is represented using probabilistic models. Several solutions to SLAM involve finding an appropriate representation for the observation model and motion model while preserving efficient and consistent computation time. Most methods use additive Gaussian noise to handle the uncertainty which imposes using extended Kalman Filter (EKF) to solve the SLAM problem (Davison, 2003). In case of using visual features, computation time and used resources grows significantly for large environments. For a complete review for SLAM methods we refer the reader to (Bailey and Durrant-Whyte, 2006).

From another side, visual odometry methods uses structure from motion methodology to estimate the relative motion (Nistér et al., 
2004). Based on multiple view geometry fundamentals (Hartley and Zisserman, 2003), approximate relative pose can be estimated, this is followed by a BA procedure to minimize reprojection errors, which yields in improving the estimated structure. Fast and efficient BA approaches are proposed simultaneously to handle larger number of images (Lourakis and Argyros, 2009). However, in case of long time navigation, the number of images increases dramatically and prevent applying global BA if real time performance is needed. Hence, several local BA approaches have been proposed to handle this problem. In local BA, a sliding window copes with motion and select a fixed number of frames to be considered for BA (Mouragnon et al., 2009). This approach does not suit S-Type motion commonly used in surveys since the last $n$ frames to the current frame are not necessarily the closest. Another local approach is the relative BA proposed in (Sibley et al., 2009). Here, the map is represented as Riemannian manifold based graph with edges representing the potential connections between frames. The method selects the part of the graph where the BA will be applied by forming two regions, an active region that contains the frames with an average re-projection error changes by more than a threshold, and a static region that contains the frames that have common measurements with frames in active region. When performing BA, the static region frames are fixed whereas active region frames are optimized. The main problem with this method is that distances between frames are metric, whereas the uncertainty is not considered when computing inter-frames distances.

Recently, a novel relative BA method is proposed by (Nawaf et al., 2016). Particularly, an approximation of the uncertainty for each estimated relative pose is estimated using a machine learning approach manifesting on simulated data. Neighboring observations used for the semi-global optimization are established based on a probabilistic distance in the estimated trajectory map. This helps to find the frames with potential overlaps with the current frame while being robust to estimation drifts. We found this method most adapted to our context.

\section{HARDWARE PLATFORM}

As mentioned earlier, we use an embedded system platform for our implementation. Being increasingly available and cheap, we choose the popular Raspberry $\mathrm{Pi}$ (C) (RPi) as main processing unit of our platform, which is a credit-card size ARM architecture based computer running Rasbain (C), a Linux variant operating system. Having 1.2 GHz 64-bit quad-core CPU and 1GB of memory allows to run smoothly most of image processing and computer vision techniques. A description of the built system is shown in Figure 2, which is composed of two RPi's computers each is connected to one camera module to form a stereo pair. The cameras are synchronized using a hardware trigger. Both computers are connected to one more powerful computer that can be either within the same enclosure or on-board in our case. Using this configuration, the embedded computers are responsible for image acquisition. The captured stereo images are first partially treated on the fly to provide image quality information as will be details in Section 4. images are then transferred to the main computer which handles the ego-motion computation that the system undergoes. For visualization purposes, we use two monitors connected to the embedded computers to show live navigation and image quality information (See Figure 3).

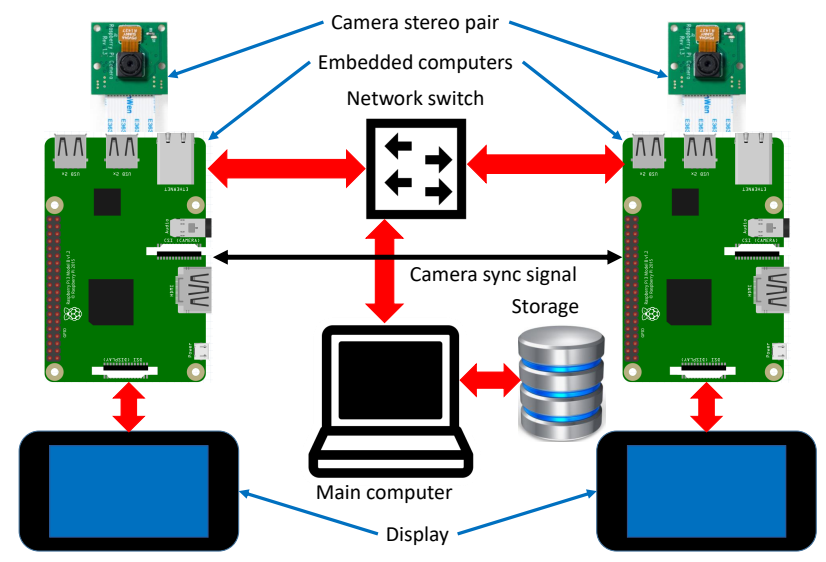

Figure 2. System hardware architecture

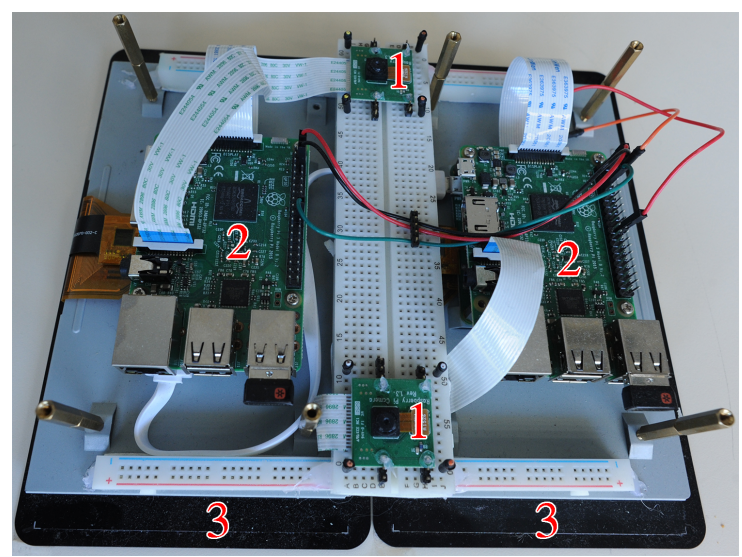

Figure 3. The hardware platform used for image acquisition and real-time navigation; it is composed mainly of (1) stereo camera pair, (2) Raspberry Pi (c) computers and (3) monitors.

\section{IMAGE QUALITY ESTIMATION}

Since underwater images do not tend to be at best conditions, a failing scenario in computing the ego-motion is expected and has to be considered. Here, we could encounter two cases; First, when having degenerated configuration that causes a failure in estimating the relative motion, this can be due to the bad image quality (blurred, dark or overexposed), the lack of textured areas or large camera displacements. That may raise ill-posed problems at several stages. Second, an imprecise estimation of the relative motion due to poorly distributed feature points or the dominant presence of outliers in the estimation procedure. As the first failure case can be straightly identified by mathematical analysis, the detection of the second case is not trivial. Nevertheless, small errors are to be corrected later using the BA procedure.

A real-time image quality estimation provides two benefits, first, it can alert the visual odometry process of having bad image quality, two reactions can be taken in this case, either pausing the process until taken image quality goes above certain threshold, or producing position estimation based on previous poses and speed. We go for the first case while leaving the second for further development in future. Second, image quality indicator provides direct information to the operator to avoid going too fast in case of blur, or changing the distance to the captured scene when going under or over-exposed. 
To estimate image sharpness, we rely on an image gradient measure that detects high frequencies often associated with sharp images, hence, we use a Sobel kernel based filtering which computes the gradient with smoothing effect. This removes the effect of dust commonly present in underwater imaging. Given an image I, first we compute

$$
\mathbf{G}=\sqrt{\left(S K^{\top} * \mathbf{I}\right)^{2}+\left(K S^{\top} * \mathbf{I}\right)^{2}}
$$

$$
\begin{array}{ll}
\text { where } & S=\left[\begin{array}{lll}
1 & 2 & 1
\end{array}\right]^{\top} \\
& K=\left[\begin{array}{lll}
-1 & 0 & 1
\end{array}\right]^{\top}
\end{array}
$$

Next, we consider the sharpness measure to be the mean value of the computed gradient magnitude image $\mathbf{G}$. The threshold can be easily learned from images by recording the number of matched feature points per image as well as image sharpness indicator. By fixing the minimum number of matched feature points needed to estimate correctly the ego-motion we can compute the minimum sharpness indicator threshold (In our experiments we fix the number of matches to 100 matches, the obtained threshold is 20). It worth to mention that several assumptions used in our work including this measure does not hold for other above-water imaging. The seabed texture guaranties a minimum sharpness even in objects-free scenes, unlike modern above-water scenes.

As mentioned earlier, image lighting conditions play an important role in computing precise odometry. Similar to image sharpness indicator, an image lightness indicator can be integrated in the odometry process as well as helping the operator to take proper actions. To estimate lightness indicator, we convert the captured images to CIE-XYZ color space and then to CIE-LAB color space. We consider the lightness indicator as the mean value of the $L$ channel. The threshold is computed in same way as for the sharpness indicator.

\section{VISUAL ODOMETRY}

After computing and displaying image quality measures, the images are transfered over the network to a third computer as shown in Figure 2. This computer is responsible for hosting the visual odometry process, which will be explained in this section. We start fist by introducing the used feature matching approach and then we present the ego-motion estimation from image quadruplets.

\subsection{Sparse Stereo Matching}

Matching feature points between stereo image pairs is essential to estimate the ego-motion. As the two cameras alignment is not perfect, we start by calibrating the camera pair. Hence, for a given point on the right image we can compute the epipolar line containing the corresponding point in the left image. However, based on the known fixed geometry, the corresponding point position is constrained by a positive disparity. Moreover, given that at deep water the only light source is the one used in our system, the most far distance that feature points can be detected is limited, see Figure 5 for illustration. This means that there is a minimum disparity value that is greater than zero. Furthermore, when going too close to the scene, parts of the image will become overexposed, similar to the previous point, this imposes a limited maximum disparity. Figure 4 illustrates the aforementioned constraints by dividing the epipolar line into 4 zones in which one is an acceptable disparity range. This range can be straightforward identified by learning from a set of captured images (oriented at 30 degrees for better coverage).

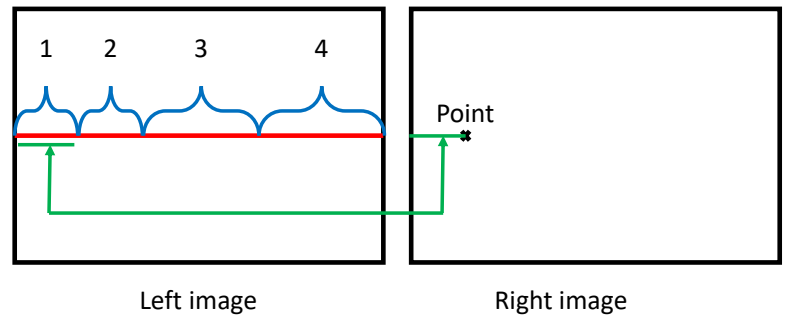

Figure 4. Illustration of stereo matching search ranges. (1) Impossible (2) Impossible in deep underwater imaging due to light's fading at far distances, where features are undetectable (3) Possible disparity (4) The point is very close so it becomes overexposed and undetectable.

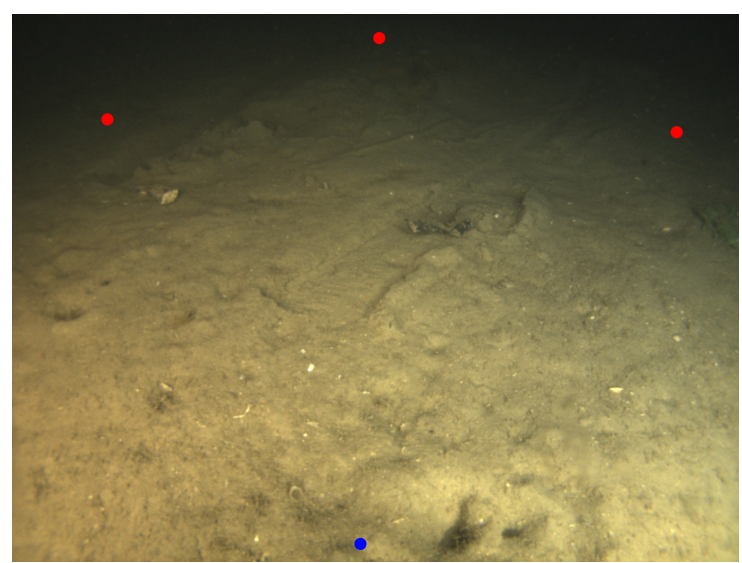

Figure 5. An example of underwater image showing minimum possible disparity (red dots, $\sim 140$ pixels) and maximum possible disparity (blue dot, $\sim 430$ pixels).

In our approach, we propose to constraint the so-called acceptable disparity range further, which corresponds to the range 3 in Figure 4. Given the used lighting system, we can assume a light diffuse reflection model where the light reflects equally in all directions. Based on inverse-square law that relates light intensity over distance, image pixels intensities are roughly proportional to their squared disparities. Based on such an assumption we could use feature point's intensity to constraint the disparity and hence limiting the range of searching for a correspondence. In order to do so, we are based on a dataset of stereo images. For each pair we perform feature points matches. Each point match $\left(x_{i}, y_{i}\right)$ and $\left(x_{i}^{\prime}, y_{i}^{\prime}\right), x$ being the coordinate in the horizontal axis, we compute the squared disparity $d_{i}^{2}=\left(x_{i}-x_{i}^{\prime}\right)^{2}$. Next, we associate each $d_{i}^{2}$ to the mean lightness value for a window centered at the given point and has a size of $2 n+1$ computed form $L$ channel in CIE-LAB color space of the right image as follows:

$$
\bar{l}_{x_{i}, y_{i}}=\frac{1}{4 n^{2}+4 n+1} \sum_{j=-n}^{n} \sum_{i=-n}^{n} L\left(x_{i}, y_{i}\right)
$$

We assign a large value $n=6$ to compensate for using Harris operator that promotes local minimum intensity pixels as salient feature points. The computed $\left(\bar{l}_{x_{i}, y_{i}}, d_{i}^{2}\right)$ pair shows the linear relationship between the squared disparity and the average light- 
ness. A subset of such pairs is plotted in Figure 6.

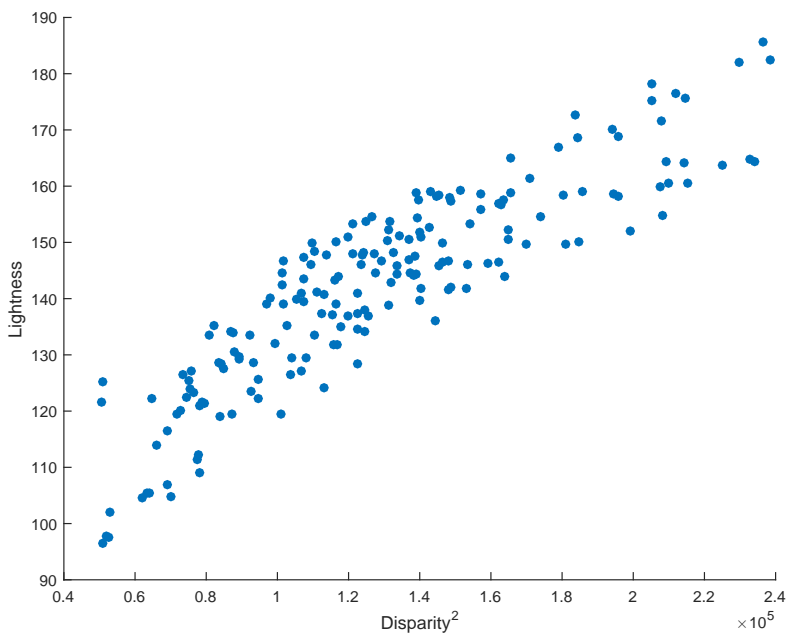

Figure 6. A subset of matched points squared disparity plotted against average pixel lightness.

In addition to finding the linear relationship between both variables that allows to give a disparity value for each lightness value, it is also necessary to capture the covariance that represents how rough is our depth approximation. More specifically, given the diagram shown in Figure 7, we aim at defining a tolerance $t$ associated to each disparity as a function of lightness $l$. In our method, we rely on Principal Component Analysis (PCA) technique to obtain this information. In details, for a given lightness $l_{i}$, we first compute the corresponding squared disparity $d_{i}^{2}$ using a linear regression approach as follows:

$$
\begin{aligned}
d_{i}^{2} & =-\alpha l_{i}-\beta \\
\alpha & =\frac{\operatorname{Cov}\left(L, D^{2}\right)}{\operatorname{Var}(L)} \\
\beta & =\bar{l}-\alpha \bar{d}^{2}
\end{aligned}
$$

where $\quad D$ is the training set of disparity, $\bar{d}$ is its mean $L$ is the training set of lightness, $\bar{l}$ is its mean

Second, let $\mathbf{V}_{2}=\left(v_{2, x}, v_{2, y}\right)$ be the computed eigenvector which correspondences to the smallest eigenvalue $\lambda_{2}$. Based on the illustration shown in Figure 7, the tolerance $t$ associated to $d_{i}^{2}$ can be written as:

$$
t=\sqrt{\lambda_{2}^{2}\left(\frac{v_{2, x}^{2}}{v_{2, y}^{2}}+1\right)}
$$

By considering a normal error distribution of the estimated rough depth, and based on the fact that $t$ is equal to one variance of $D^{2}$, we define the effective disparity range as:

$$
d_{i} \pm \gamma \sqrt[4]{t}
$$

where $\gamma$ represents the number of standard deviations. It is trivial that $\gamma$ is a trade-off between computation time and the probability of having points correspondences within the chosen tolerance

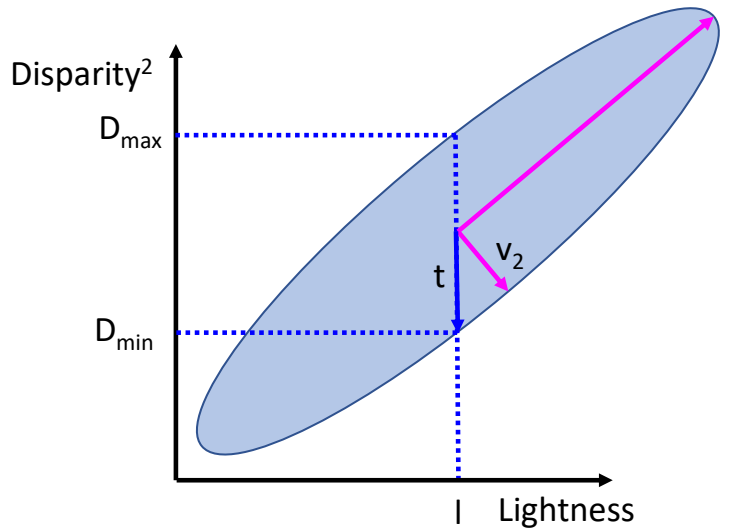

Figure 7. Illustration of disparity tolerance $t$ given a lightness value $l$.

range. We set $\gamma=2$ which means there is $95 \%$ probability to cover the data.

At this step, we have all the information needed to proceed for the points matching. In which for each feature point in the right image, we search for the corresponding point in the left image based on a sliding window over a rectangle whose width is defined by the Equations 3 to 7, and the height is defined empirically to allow for distortion and stereo misalignment. After testing several distance measures, we found that normalized measures tend to be more robust to underwater imaging. In particular, we use the normalized cross correlation computed as:

$$
R(x, y)=\frac{\sum_{x^{\prime}, y^{\prime}}\left(W_{r}\left(x^{\prime}, y^{\prime}\right) \cdot I_{l}\left(x+x^{\prime}, y+y^{\prime}\right)\right)}{\sqrt{\sum_{x^{\prime}, y^{\prime}} W_{r}\left(x^{\prime}, y^{\prime}\right)^{2} \cdot \sum_{x^{\prime}, y^{\prime}} I_{l}\left(x+x^{\prime}, y+y^{\prime}\right)^{2}}}
$$

where $W_{r}$ is the sliding window in the right image centered at the query pixel. $I_{l}$ is the left image. $R$ is the response that we seek to maximize.

\subsection{Ego-Motion Estimation}

Since the stereo cameras are already calibrated, their relative position is known in advance. Both cameras have the same intrinsic parameters matrix $K$. Given left and right frames at time $t$ (we call them previous frames), our visual odometry pipeline consists of four stages:

- Feature points detection and matching for every new stereo pair $t+1$.

- 3D reconstruction of the matched feature points using triangulation.

- Relative motion computation using adaptation between the point clouds for the frames at $t$ and $t+1$.

- Relative BA procedure is applied to minimize re-projection errors;

In details, let $\left(f_{1}, f_{2}, f_{3}, f_{4}\right)$ denote the previous left, previous right, current left and current right frames respectively. For each new captured image pair, we compute a 3D point cloud using triangulation as described in (Hartley and Zisserman, 2003) for the matched feature points that are obtained using the method proposed in the previous subsection. 
The rigid transformation $[R \mid T]$ required for expressing the frames at time $t+1$ in the reference frame at time $t$ is the rigid transformation required to move the $3 \mathrm{D}$ point cloud at time $t$ to the one obtained at time $t+1$. Hence, the problem of calculating the orientation of the cameras at time $t+1$ in relation to time $t$ leads back to the calculation of the transformation used to move from one point cloud to the other. This is possible under our configuration, with small rotation. We note here that there is no scale problem between both point clouds which is specific to stereo systems. We consider here the left previous to left current frames $f_{1} \rightarrow f_{3}$ positions to represent the system relative motion, and their relative transformation denoted $\left[R_{13} \mid T_{13}\right]$.

Below, we present the method to compute the transformation for passing from the point cloud calculated at time $t+1$, denoted $P$, to the one calculated at time $t$, denoted $P^{\prime}$. So we have two sets of $n$ homologous points $P=P_{i}$ and $P^{\prime}=P_{i}^{\prime}$ where $1 \leq i \leq n$. We have:

$$
P_{i}^{\prime}=R_{13} P_{i}+T_{13}
$$

The best transformation the minimizes the error $r$, the sum of the squares of the residuals:

$$
r=\sum_{i=1}^{n}\left\|R_{13} P_{i}+T_{13}-P_{i}^{\prime}\right\|^{2}
$$

To solve this problem, we use the singular value decomposition (SVD) of the covariance matrix $\mathrm{C}$ :

$$
r=\sum_{i=1}^{n}\left(P_{i}-\bar{P}\right)\left(P_{i}^{\prime}-\bar{P}^{\prime}\right)
$$

where $\bar{P}$ and $\bar{P}^{\prime}$ are the centers of mass of the 3D points sets $P$ and $P^{\prime}$ respectively. Given the SVD of $\mathrm{C}$ as: $[U, S, V]=$ $S V D(C)$, the final transformation is computed as:

$$
\begin{aligned}
R_{13} & =V U^{\top} \\
T_{13} & =-R_{13} \bar{P}+\bar{P}^{\prime}
\end{aligned}
$$

Once the image pair $t+1$ is expressed in the reference system of the image pair $t$, the $3 \mathrm{D}$ points can be recalculated using the four observations that we have for each point. A set of verifications are then performed to minimize the pairing errors (verification of the epipolar line, the consistency of the y-parallax, and re-projection residues). Once validated, the approximated camera position at time $t+1$ are used as input values for the $\mathrm{BA}$ as described earlier.

\section{EVALUATION}

We implemented our method using OpenCV library and SBA toolbox proposed by (Lourakis and Argyros, 2009). Since our main goal is to reduce the processing time, we tested and compared the computation speed of our method compared to using high level feature descriptors, specifically SIFT and SURF. At the same time, we monitor the precision for each test. The evaluation is done using the same set of images.

In the obtained results, the computation time when using the reduced matching search range as proposed in this work is $\sim 72 \%$

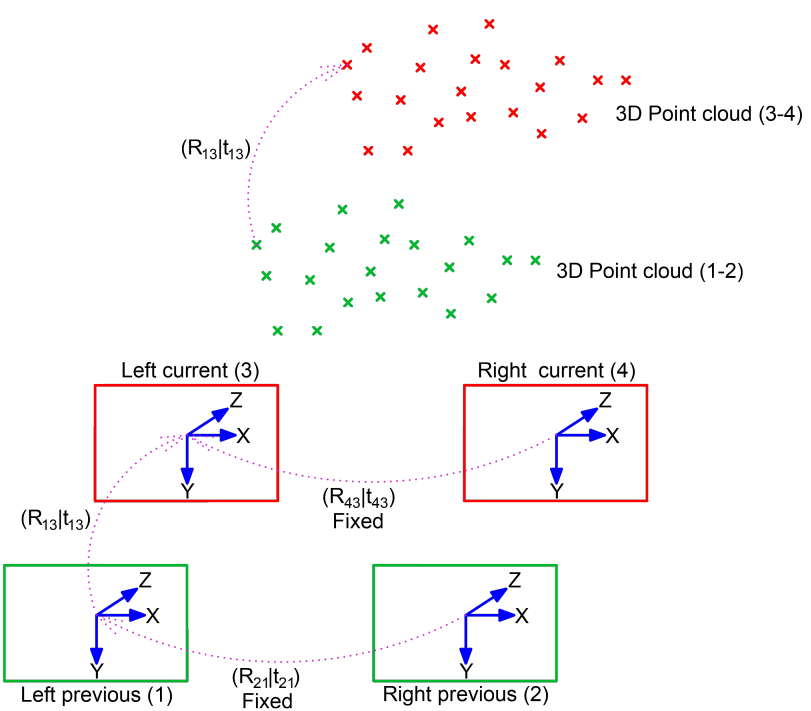

Figure 8. Image quadruplet, current (left and right) and previous (left and right) frames are used to compute two 3D point clouds. The transformation between the two points clouds is equal to the relative motion between the two camera positions.

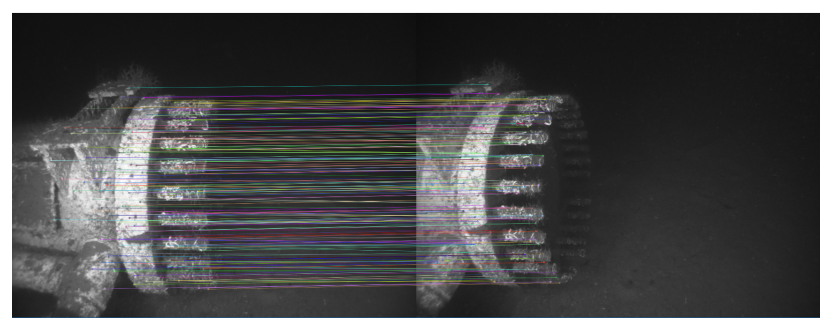

Figure 9. An example of stereo matching using the proposed method

compared $^{1}$ to the method using the whole search range (range 3 in Figure 4). Concerning SIFT and SURF, the computation time is $342 \%$ and $221 \%$ respectively compared to the proposed method. The precision of the obtained odometry is reasonable which is within the limit of $3 \%$ for the average translational error and $0.02[\mathrm{deg} / \mathrm{m}]$ for the average rotational error.

\section{CONCLUSIONS AND PERSPECTIVES}

In this work, we introduced several improvements to the current traditional visual odometry approach in order to serve in the context of underwater surveys. The goal is to be adapted to embedded systems known for their lower resources. We resume the developed processing pipeline applied to each captured stereo image pair as follows: A sharpness indicator is computed based on quantifying image frequencies, and a lightness indicator is computed using CIELAB colour space, both indicators help the operator during image acquisition. A sparse feature points detection and improved matching is proposed. The matching is guided with a rough depth estimation using lightness information, this is the factor beyond most of the gain in computation time com-

\footnotetext{
${ }^{1}$ The time evaluation is shown in percentage because the evaluation is carried out on three platforms with different computational power, in which one is an embedded unit. The minimum computation time being $220 \mathrm{~ms}$
} 
pared to sophisticated feature descriptors combined with bruteforce matching.

The evaluation of our system is ongoing. A deep-sea mission is planned in short term in corporation with COMEX $^{2}$ our industrial partner that provides needed instrumental infrastructure.

Our future perspectives are mainly centered on reducing the overall system size, for instance, replacing the main computer in our architecture with a third embedded unit, which in turn does not keep evolving. This also allows to reduce the power consumption which increases the navigation time. On the other hand, dealing with visual odometry failure is an important challenge specially in the context of underwater imaging, which is mainly due to bad image quality. The ideas of failing scenarios discussed in this paper can be extended to deal with the problem of interruptions in the obtained trajectory.

\section{ACKNOWLEDGEMENTS}

This work has been partially supported by both a public grant overseen by the French National Research Agency (ANR) as part of the program Contenus numériques et interactions (CONTINT) 2013 (reference: ANR-13-CORD-0014), GROPLAN project (Ontology and Photogrammetry; Generalizing Surveys in Underwater and Nautical Archaeology) ${ }^{3}$, and by the French Armaments Procurement Agency (DGA), DGA RAPID LORI project (LOcalisation et Reconnaissance d'objets Immergés).

\section{REFERENCES}

Bailey, T. and Durrant-Whyte, H., 2006. Simultaneous localization and mapping (slam): Part ii. IEEE Robotics \& Automation Magazine 13(3), pp. 108-117.

Bay, H., Tuytelaars, T. and Van Gool, L., 2006. Surf: Speeded up robust features. European Conference on Computer Vision pp. 404-417.

Davison, A. J., 2003. Real-time simultaneous localisation and mapping with a single camera. In: Computer Vision, 2003. Proceedings. Ninth IEEE International Conference on, IEEE, pp. 1403-1410.

Drap, P., 2012. Underwater photogrammetry for archaeology. INTECH Open Access Publisher.

Gauglitz, S., Höllerer, T. and Turk, M., 2011. Evaluation of interest point detectors and feature descriptors for visual tracking. International journal of computer vision 94(3), pp. 335-360.

Geiger, A., Ziegler, J. and Stiller, C., 2011. Stereoscan: Dense 3d reconstruction in real-time. In: IEEE Intelligent Vehicles Symposium, pp. 963-968.

Hartley, R. and Zisserman, A., 2003. Multiple view geometry in computer vision. Cambridge university press.

Kitt, B., Geiger, A. and Lategahn, H., 2010. Visual odometry based on stereo image sequences with ransac-based outlier rejection scheme. In: Intelligent Vehicles Symposium (IV), 2010 IEEE, IEEE, pp. 486-492.

Lourakis, M. I. and Argyros, A. A., 2009. Sba: A software package for generic sparse bundle adjustment. ACM Transactions on Mathematical Software 36(1), pp. 2.

\footnotetext{
${ }^{2}$ http: //www . comex.fr/

${ }^{3}$ http: //www.groplan.eu
}

Lowe, D., 2004. Distinctive image features from scale-invariant keypoints. International Journal of Computer Vision 60(2), pp. 91-110.

Montemerlo, M. and Thrun, S., 2007. FastSLAM: A scalable method for the simultaneous localization and mapping problem in robotics. Vol. 27, Springer.

Mouragnon, E., Lhuillier, M., Dhome, M., Dekeyser, F. and Sayd, P., 2009. Generic and real-time structure from motion using local bundle adjustment. Image and Vision Computing 27(8), pp. 1178-1193.

Nawaf, M. M. and Trémeau, A., 2014. Monocular 3d structure estimation for urban scenes. In: Image Processing (ICIP), 2014 IEEE International Conference on, IEEE, pp. 3763-3767.

Nawaf, M. M., Hijazi, B., Merad, D. and Drap, P., 2016. Guided underwater survey using semi-global visual odometry. 15th International Conference on Computer Applications and Information Technology in the Maritime Industries pp. 288-301.

Nistér, D., Naroditsky, O. and Bergen, J., 2004. Visual odometry. In: Computer Vision and Pattern Recognition, 2004. CVPR 2004. Proceedings of the 2004 IEEE Computer Society Conference on, Vol. 1, IEEE, pp. I-652.

Sibley, D., Mei, C., Reid, I. and Newman, P., 2009. Adaptive relative bundle adjustment. In: Robotics: science and systems, Vol. 32, p. 33.

Thrun, S., Burgard, W. and Fox, D., 2005. Probabilistic robotics. MIT press.

Triggs, B., McLauchlan, P., Hartley, R. and Fitzgibbon, A., 2000. Bundle adjustment: a modern synthesis. Vision algorithms: theory and practice pp. 153-177.

Yamaguchi, K., McAllester, D. and Urtasun, R., 2013. Robust monocular epipolar flow estimation. In: Proceedings of IEEE Conference on Computer Vision and Pattern Recognition, CVPR '13, IEEE Computer Society, Washington, DC, USA, pp. 18621869. 